\title{
X-ray wind tomography of the highly absorbed HMXB IGR J17252-3616`
}

\author{
A. Manousakis ${ }^{1,2}$ and R. Walter ${ }^{1,2}$ \\ ${ }^{1}$ ISDC Data Center for Astrophysics, Université de Genève, Chemin d'Ecogia 16, 1290 Versoix, Switzerland \\ e-mail: Antonios. Manousakis@unige.ch \\ 2 Observatoire de Genève, Université de Genève, Chemin des Maillettes 51, 1290 Versoix, Switzerland
}

Received 7 September 2010 / Accepted 6 November 2010

\begin{abstract}
Context. About ten persistently highly absorbed super-giant high-mass X-ray binaries (sgHMXB) have been discovered by INTEGRAL as bright hard X-ray sources lacking bright X-ray counterparts. Besides IGR J16318-4848, which has peculiar characteristics, the other members of this family share many properties with the classical wind-fed sgHMXB systems.

Aims. Our goal is to understand the characteristics of highly absorbed sgHMXB and in particular the companion stellar wind, which is thought to be responsible for the strong absorption.

Methods. We monitored IGR J17252-3616, a highly absorbed system featuring eclipses, with XMM-Newton to study the variability of the column density and the Fe K $\alpha$ emission line along the orbit and during the eclipses. We also compiled a 3D model of the stellar wind to reproduce the observed variability.

Results. We first derive a refined orbital solution based on INTEGRAL, RXTE, and XMM-Newton data. We find that the XMM-Newton monitoring campaign reveals significant variations in the intrinsic absorbing column density along the orbit and the Fe K $\alpha$ line equivalent width around the eclipse. The origin of the soft X-ray absorption is associated with a dense and extended hydrodynamical tail, trailing the neutron star. This structure extends along most of the orbit, indicating that the stellar wind has been strongly disrupted. The variability of the absorbing column density suggests that the wind velocity is smaller $\left(v_{\infty} \approx 400 \mathrm{~km} \mathrm{~s}^{-1}\right)$ than observed in classical systems. This may also explain the much stronger density perturbation inferred from the observations. Most of the Fe K $\alpha$ emission is generated in the innermost region of the hydrodynamical tail. This region, which extends over a few accretion radii, is ionized and does not contribute to the soft X-ray absorption.

Conclusions. We present a qualitative model of the stellar wind of IGR J17252-3616 that can represent the observations, and we suggest that highly absorbed systems have lower wind velocities than classical sgHMXB. This proposal could be tested with detailed numerical simulations and high-resolution infrared/optical observations. If confirmed, it may turn out that half of the persistent sgHMXB have low stellar wind speeds.
\end{abstract}

Key words. X-rays: binaries - stars: winds, outflows - pulsars: individual: IGR J17252-3616 - supergiants

\section{Introduction}

High mass X-ray binaries (HMXB) consist of a neutron star or a black hole fueled by the accretion of the wind of an early-type stellar companion. Their X-ray emission, a measure of the accretion rate, shows a variety of transient to persistent patterns. Outbursts are observed on timescales from seconds to months and dynamical ranges varying by factors of $10^{4}$. The majority of the known HMXB are Be/X-ray binaries (Liu et al. 2006), with Be stellar companions. These systems are transient, featuring bright outbursts with typical durations on the order of several weeks (White 1989; White et al. 1995; Charles \& Coe 2006). A second class of HMXBs harbor OB supergiant companions (sgHMXBs) that feed the compact object by means of strong, radiatively driven stellar winds or Roche lobe overflow. Thanks to INTEGRAL, the number of known sgHMXB systems has tripled in the past few years (Walter et al. 2006).

Highly absorbed sgHMXB were discovered by INTEGRAL (Walter et al. 2004) and are characterized by strong and

* Based on observations obtained with XMM-Newton and INTEGRAL, two ESA science mission with instruments, data centers, and contributions directly funded by ESA Member States, NASA, and Russia. persistent soft X-ray absorption $\left(N_{\mathrm{H}}>10^{23} \mathrm{~cm}^{-2}\right)$. When detected, these systems have short orbital periods and long spin periods (Walter et al. 2006). They correspond to the category of wind-fed accretors in the Corbet diagram (Corbet 1986).

IGR J17252-3616 was detected by ISGRI onboard INTEGRAL on February 9, 2004 among other hard X-ray sources (Walter et al. 2004, 2006). The source was first detected by EXOSAT (EXO 1722-3616) as a weak soft X-ray source, back in 1984 (Warwick et al. 1988). In 1987, Ginga performed a pointed observations and revealed a highly variable X-ray source, X1722-363, with a pulsation period of $\sim 413.9 \mathrm{~s}$ (Tawara et al. 1989). Additional Ginga observations revealed the orbital period of 9-10 days and a mass of the companion star of $\sim 15 M_{\odot}$ (Takeuchi et al. 1990). Both papers concluded that the system was a high mass X-ray binary (HMXB).

INTEGRAL and XMM - Newton observations of IGR J17252-3616 allowed Zurita Heras et al. (2006) to identify the infrared counterpart of the system, to accurately measure the absorbing column density, and refine the spin period of the system. Thanks to the eclipses, an accurate orbital period could be derived from INTEGRAL data. Further RXTE observations helped identify a highly inclined system $\left(i>61^{\circ}\right)$ with a companion star of $M_{*} \lesssim 20 M_{\odot}$ and $R_{*} \sim 20-40 R_{\odot}$ 
Table 1. XMM-Newton observation log.

\begin{tabular}{lcccccc}
\hline \hline ObsID & $\#$ & Revolution & Start time (UT) & $\begin{array}{c}\text { Effective } \\
\text { exposure }(\mathrm{ks})\end{array}$ & Source counts & $\begin{array}{c}\text { Phase } \\
( \pm 0.01)\end{array}$ \\
\hline 0405640201 & 1 & 1231 & 2006-08-29T03:02:58 & 19.2 & $5.4 \times 10^{2}$ & 0.03 \\
0405640301 & 2 & 1232 & 2006-08-31T16:37:44 & 4.1 & $1.4 \times 10^{4}$ & 0.27 \\
0405640401 & 3 & 1234 & 2006-09-04T06:35:33 & 5.6 & $7.9 \times 10^{3}$ & 0.65 \\
0405640501 & 4 & 1235 & 2006-09-06T19:33:10 & 5.4 & $9.5 \times 10^{2}$ & 0.91 \\
0405640601 & 5 & 1236 & 2006-09-08T10:03:38 & 7.9 & $3.8 \times 10^{2}$ & 0.08 \\
0405640701 & 6 & 1239 & 2006-09-15T07:23:45 & 2.8 & $1.7 \times 10^{3}$ & 0.79 \\
0405640801 & 9 & 1247 & 2006-10-01T03:24:26 & 9.4 & $2.3 \times 10^{4}$ & 0.40 \\
0405640901 & 8 & 1246 & 2006-09-28T14:36:53 & 11.3 & $1.9 \times 10^{4}$ & 0.15 \\
0405641001 & 7 & 1245 & 2006-09-27T07:27:58 & 9.4 & $6.5 \times 10^{2}$ & 0.01 \\
\hline 0206380401 & 10 & 785 & 2004-03-21T13:23:09 & 8.6 & $4.7 \times 10^{4}$ & 0.37 \\
\hline
\end{tabular}

Notes. The revolution number, the start time, the effective exposure, the source counts, and the phase, calculated at the middle of each observation using the orbital solution obtained with fixed orbital period (table 3 are shown.

(Thompson et al. 2007). Recent VLT observations help us to infer the companion spectral type (Chaty et al. 2008; Mason et al. 2009) and radial velocity measurements (Mason et al. 2010). Its spectral energy distribution can be characterized by a temperature of $T_{*} \sim 30 \mathrm{kK}$ and a reddening of $A_{\mathrm{V}} \sim 20$ (Rahoui et al. 2008).

In this paper, we report on a monitoring campaign of IGR J17252-3616 performed with XMM-Newton along the orbit in order to estimate the structure of the stellar wind and the absorbing material in the system. We describe the data and their analysis in Sect. 2 and a refined orbital solution in Sect. 3, and we present the evolution of the X-ray spectrum during the orbit in Sect. 4. In Sect. 5, we present and discuss a 3D model of the stellar wind that can reproduce the observations and present our conclusions in Sect. 6.

\section{Data reduction and analysis}

\subsection{XMM-Newton}

Pointed observations of IGR J17252-3616 were performed between August and October 2006 with XMM-Newton (Jansen et al. 2001). We scheduled 9 observations to cover the orbital phases $0.01,0.03,0.08,0.15,0.27,0.40,0.65,0.79$, and 0.91 . In addition, we used one observation of 2004 with a phase of 0.37 . The observations are summarized in Table 1 .

The Science Analysis Software (XMM-SAS) version 9.0.0 was used to produce event lists for the EPIC-pn instrument (Strüder et al. 2001) by running epchain. Barycentric correction and good time intervals (GTI) were applied. Photon pileup and/or out-of-time events were not identified among the data. High level products (i.e., spectra and lightcurves) were produced using evselect ${ }^{2}$. Spectra and lightcurves were built by collecting double and single events in the energy range $0.2-10 \mathrm{keV}$. The lightcurves were built using $5 \mathrm{~s}$ time bins. The spectra were re-binned to obtain 25 counts/bin for low count-rate observations and 100 counts/bin for high count-rate observations.

In one dataset (ObsID 0405640701, $\sim 19 \mathrm{ks}$ ), the count rate above $10 \mathrm{keV}$ has a very peculiar behavior, increasing monotonically with time. As this does not affect the background subtracted source lightcurve significantly, we used the entire set of

\footnotetext{
1 http://xmm.esac.esa.int/sas/

2 http://xmm. esac. esa.int/sas/current/howtousesas. shtml
}

data in the pulse arrival-time determination. Standard GTI was used for spectral analysis.

\subsection{INTEGRAL}

We analyzed the hard X-ray lightcurve of IGR J17252-3616 obtained with ISGRI (Lebrun et al. 2003) on board INTEGRAL (Winkler et al. 2003). We extracted the 22-40 keV lightcurve using the HEAVENS ${ }^{3}$ interface (Walter et al., in prep.). The lightcurve includes all data available on IGR J17252-3616 from Jan. 29, 2003 06:00:00 to Apr. 8, 2009 00:28:48 UTC. The effective exposure time on source is $\sim 3.6 \mathrm{Ms}$.

\section{Timing analysis and orbit determination}

\subsection{Orbital period from INTEGRAL}

We used the Lomb-Scargle (Press \& Rybicki 1989) technique to determine the orbital period from the INTEGRAL light-curve and obtained $P_{\text {orb }}=9.742 \pm 0.001$ days. Figure 1 shows (upper panel) the Lomb-Scargle power around the orbital period (dashed line). This period was used to refine the orbital solution (Sect. 3.3). The lower panel of Fig. 1 shows the lightcurve folded with the newly derived orbital period. The eclipse is clearly detected with the count rate dropping to zero.

\subsection{Pulse arrival times}

Pulse arrival times (PATs, hereafter) were obtained from the broad-band $0.2-10 \mathrm{keV}$ lightcurves obtained by XMM-Newton. Close to the eclipse $(\phi=0.03,0.08,0.91,0.01)$, when the compact object is behind the massive star, pulses could not be detected. We did not extract PATs for the observation of 2004.

To determine the PATs, we used a pulse profile template. This template is derived by folding the lightcurve from observation 0405640801 with a period of $414.2 \mathrm{~s}$, obtained using the Lomb-Scargle technique (Press \& Rybicki 1989). This observation was selected because the source was very bright for a long and almost uninterrupted exposure.

A sequence of pulse profile template was fit to each individual lightcurve. This sequence is characterized by: (i) the time of a pulse at the middle of the observation; (ii) the pulse period; and (iii) the amplitudes of each pulse. This assumes that the pulse period is reasonably constant during each observation.

\footnotetext{
${ }^{3}$ http://www . isdc. unige.ch/heavens
} 

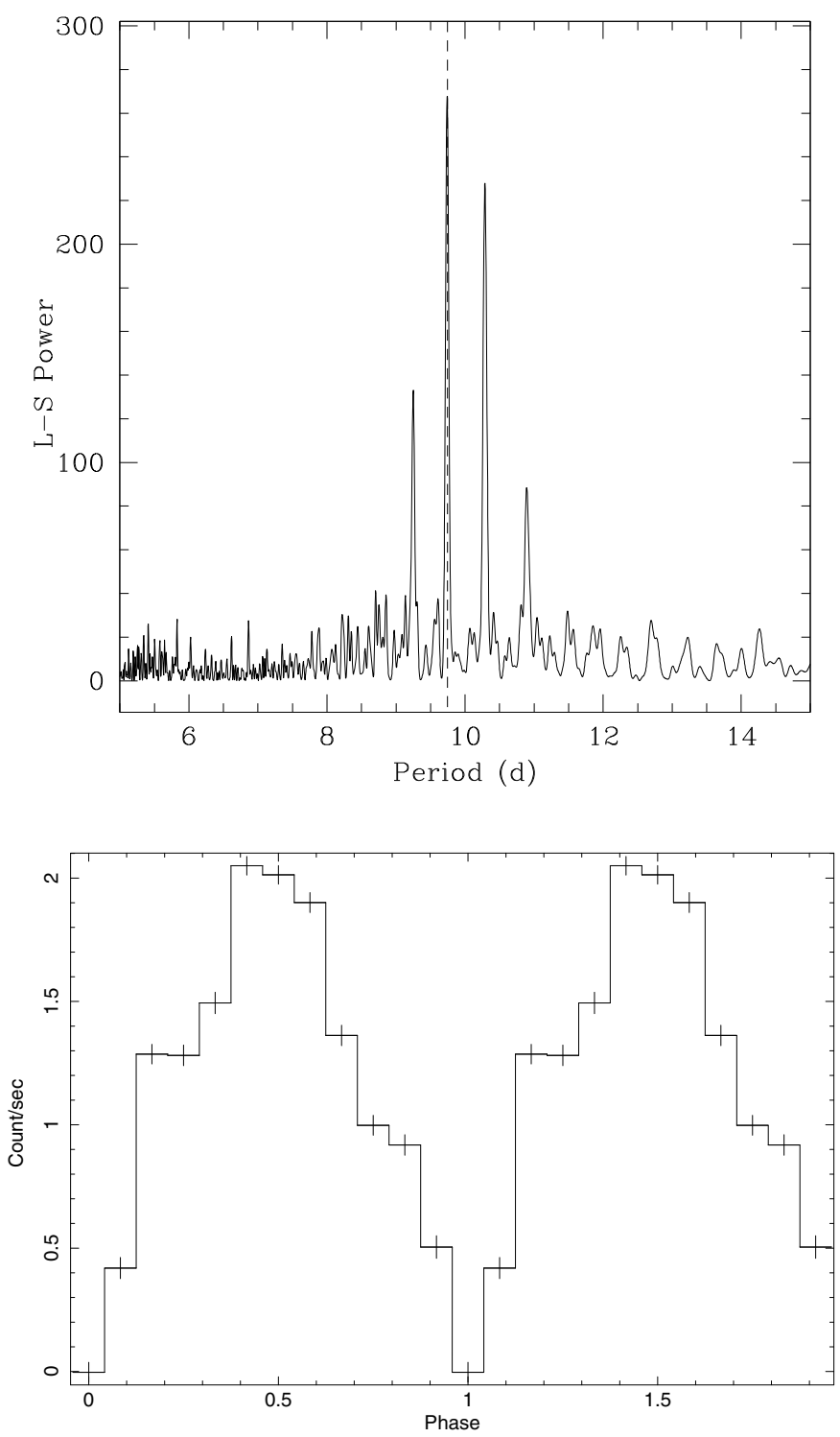

Fig. 1. Top: Lomb-Scargle periodogram obtained from the INTEGRAL 22-40 keV lightcurve. Bottom: INTEGRAL lightcurve folded with a period of 9.742 days.

Table 2. Pulse arrival times and derived pulse period.

\begin{tabular}{lcc}
\hline \hline ObsID & $\begin{array}{c}\text { PAT (HJD) } \\
( \pm 0.00011)\end{array}$ & Pulse period (s) \\
\hline 0405640301 & 53978.74948 & $414.3 \pm 0.1$ \\
0405640401 & 53982.32329 & $414.0 \pm 0.1$ \\
0405640701 & 53993.45320 & $414.2 \pm 0.2$ \\
0405640801 & 54009.24191 & $414.2 \pm 0.1$ \\
0405640901 & 54006.75737 & $413.8 \pm 0.1$ \\
\hline
\end{tabular}

The statistical errors in the pulse time and period are typically $0.01 \mathrm{~s}$ and $0.1 \mathrm{~s}$, respectively. Table 2 lists the pulse times and the pulse period obtained at the middle of each observation.

The PAT accuracy is limited by the systematic error related to the assumed pulse profile template. Using a different pulse profile template (derived from observation 0405640901) produces PAT with an offset between $8 \mathrm{~s}$ and $12 \mathrm{~s}$ from the values listed in Table 2. We adopted a systematic error of $10 \mathrm{~s}$.
Table 3. Orbital solution for IGR J17252-3616.

\begin{tabular}{lllll}
\hline \hline & Units & Free $P_{\text {orb }}$ & Fixed $P_{\text {orb }}$ & RXTE Epoch 3 \\
\hline$t_{0}^{\text {XTE }}$ & HJD & 53761.73144 & 53761.73142 & 53761.73126 \\
$P_{0}^{\mathrm{XTE}}$ & $\mathrm{s}$ & $413.889 \pm 0.004$ & $413.889 \pm 0.005$ & $413.894 \pm 0.002$ \\
$\dot{P}^{\mathrm{XTE}}$ & $\mu \mathrm{s} \mathrm{s}^{-1}$ & $-0.010 \pm 0.002$ & $-0.010 \pm 0.003$ & $-0.0106 \pm 0.0001$ \\
\hline$t_{0}^{\mathrm{XMM}}$ & $\mathrm{HJD}$ & 53978.7494 & 53978.7495 & - \\
$P_{0}^{\mathrm{XMM}}$ & $\mathrm{s}$ & $413.86 \pm 0.04$ & $413.84 \pm 0.04$ & - \\
$\dot{P}^{\mathrm{XMM}}$ & $\mu \mathrm{s} \mathrm{s}^{-1}$ & $0.98 \pm 0.04$ & $1.01 \pm 0.03$ & - \\
\hline$\alpha_{x} \sin i$ & $\mathrm{lt}-\mathrm{s}$ & $102 \pm 8$ & $101 \pm 2$ & $101 \pm 4$ \\
$P_{\text {orb }}$ & $\mathrm{d}$ & $9.76 \pm 0.02$ & $9.742($ fixed $)$ & $9.78 \pm 0.04$ \\
$T_{90}$ & $\mathrm{HJD}$ & $53761.62 \pm 0.1$ & $53761.69 \pm 0.1$ & $53761.60 \pm 0.09$ \\
\hline$\chi_{v}^{2}$ & & $1.5(30)$ & $1.65(31)$ & $1.45(28)$ \\
\hline
\end{tabular}

Notes. The errors have been calculated at $90 \%$ confidence level. The errors in the arrival times $t_{0}$ are 0.00001 and 0.0001 days for RXTE and $X M M$, respectively. The last column shows the result from Thompson et al. (2007) for comparison.

\subsection{Orbital solution}

We derived the orbital solution using the PATs of the RXTE observation obtained by Thompson et al. (2007, Epoch 3, see Fig. 2), and the PATs derived above from XMM data.

The orbital solution was obtained by comparing the observed pulse arrival delays $\left(t_{n}-t_{0}-n P_{0}-\frac{1}{2} n^{2} P_{0} \dot{P}\right)$ to the expected ones $\alpha_{x} \sin i \cos \left[2 \pi\left(t_{n}-T_{90}\right) / P_{\text {orb }}\right]$ (Levine et al. 2004). The orbital parameters (the orbital period, $P_{\text {orb }}$; the projected semi-major axis, $\alpha_{x} \sin i$; the reference time corresponding to mid-eclipse, $T_{90}$ ) were assumed to be constant. To account for pulse evolution, two sets of pulse parameters (spin period at time $t_{0}, P_{0}$; spin period derivative, $\dot{P}$ ) were used for the $R X T E$ and $X M M$ campaigns. The pulse number $n$ was given by the nearest integer to $n=\left(t_{n}-t_{0}\right) /\left(P_{0}+0.5 \dot{P}\left(t_{n}-t_{0}\right)\right)$.

We performed a combined fit of the RXTE and XMM observations. We derived the orbital solutions by (i) allowing all parameters to vary freely and (ii) fixing the orbital period to the value derived from the INTEGRAL data. The resulting parameters are listed in Table 3. We were also able to obtain an upper limit $(90 \%)$ of $e<0.15$ on the eccentricity by adding the first-order term in a Taylor series expansion in the eccentricity (Levine et al. 2004). The RXTE and XMM orbital solutions are comparable and the resulting parameters are consistent within the errors.

The folded lightcurve obtained with the INTEGRAL derived orbital period (Fig. 1, lower panel) results in a pulse fraction $\sim 100 \%$. For the rest of the analysis, we used the orbital solution obtained with fixed $P_{\text {orb }}$ (Table 3).

Figure 2 shows the resulting pulse arrival-time delays (fixed $P_{\mathrm{orb}}$ ) for both $R X T E$ and XMM data together with the best-fit orbital solution.

\section{Spectral analysis}

The spectral analysis was performed using the $\mathrm{XSPEC}^{4}$ package version 11.3.2ag (Arnaud 1996). To use the $\chi_{v}^{2}$ statistics, we grouped the data to have at least 25 (faint spectra) and up to 100 (bright spectra) counts per bin. We initially fitted the observed spectra using a phenomenological model made of an intrinsically absorbed cutoff power-law, a blackbody soft excess, and a gaussian $\mathrm{Fe} \mathrm{K} \alpha$ line (wabs*(bb+gauss+vphabs*cutoff)). The

${ }^{4}$ http://xspec.gsfc.nasa.gov 


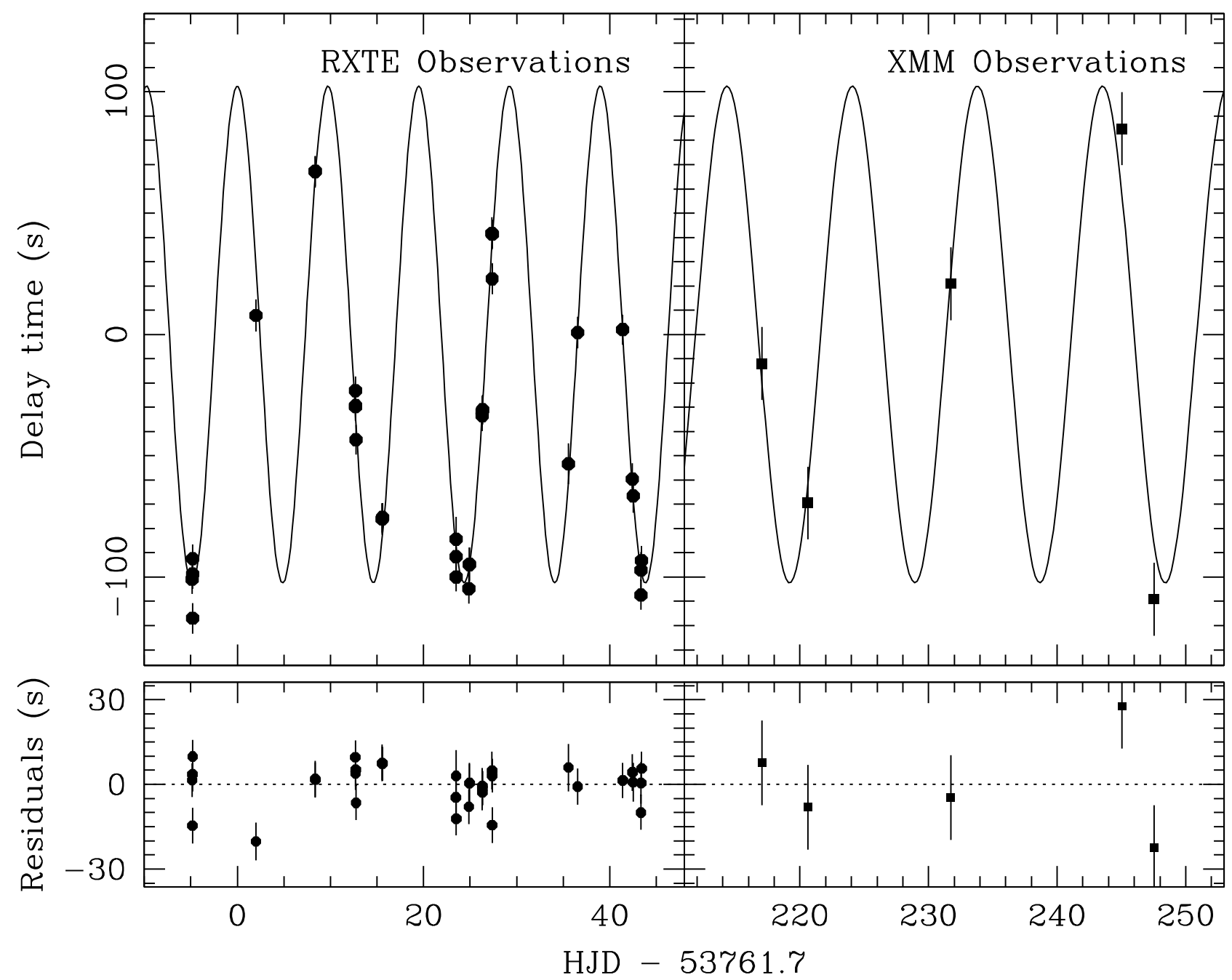

Fig. 2. Delays (top) and residuals (bottom) derived from the fixed $P_{\text {orb }}$ orbital solution compared to the data. Left: RXTE data are taken from Thompson et al. (2007). Right: XMM - Newton data from this work.

centroid of the iron line is compatible with $E_{\mathrm{c}} \sim 6.40 \pm 0.03 \mathrm{keV}$ at all epochs.

The spectra are always strongly absorbed below $\sim 3 \mathrm{keV}$ and display an iron K-edge at $\sim 7.2 \pm 0.2 \mathrm{keV}$. We first fit each spectrum by assuming all parameters to be free, apart from the Galactic absorption, which was fixed to $N_{\mathrm{H}}=1.5 \times 10^{22} \mathrm{~cm}^{-2}$ (Dickey \& Lockman 1990).

Some parameters (photon index, cutoff energy, blackbody temperature, and absorber Fe metallicity) did not vary (within the $90 \%$ errors) among the observations and were fixed to their average values of $E_{\mathrm{C}}=8.2 \mathrm{keV}, \Gamma=0.02$, and $k T_{\mathrm{BB}}=0.5 \mathrm{keV}$, $Z=1 Z_{\odot}$. The Fe line was fixed to an energy of $6.4 \mathrm{keV}$, with a narrow width. Two representative spectra are displayed in Fig. 3.

The intrinsic absorbing column density and the normalization of each component were allowed to vary freely. The bestfit model parameters are listed in Table 4 . Figure 4 shows the variation in all these parameters and in both the unabsorbed $2-10 \mathrm{keV}$ flux and $\mathrm{Fe} \mathrm{K} \alpha$ equivalent width $(E W)$. The unabsorbed $\mathrm{Fe} \mathrm{K} \alpha E W$ was calculated by setting the intrinsic absorbing column density to zero. All the spectral fits were good resulting in $\chi_{v}^{2} \approx 0.8-1.4$, apart from the $\phi=0.03$ observation for which a poor fit was obtained. For the observations made during the eclipse, some parameters are poorly constrained.

The unabsorbed continuum flux (Fig. 4f) is on the order of $\sim 5 \times 10^{-11} \mathrm{erg} \mathrm{cm}^{-2} \mathrm{~s}^{-1}$ outside the eclipse. Variations

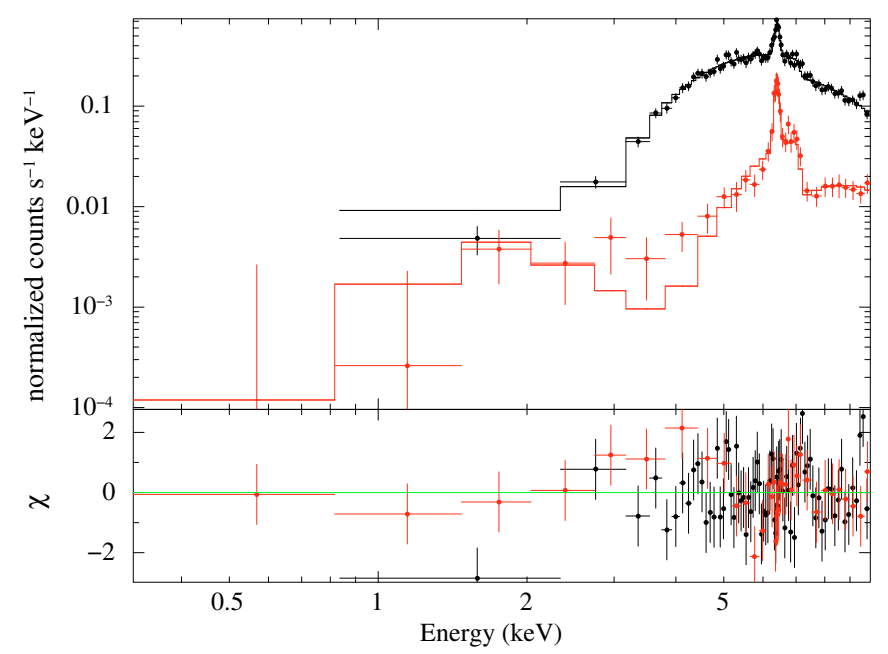

Fig. 3. Top: folded model and data for $\phi=0.65$ (black) and $\phi=0.91$ (red). Bottom: the corresponding residuals using the model described in the text with the parameters from Table 4.

are observed across the range $(2-10) \times 10^{-11} \mathrm{erg} \mathrm{cm}^{-2} \mathrm{~s}^{-1}$ and can be interpreted as variations in the accretion rate $(\dot{M})$. During the eclipse, the continuum flux drops by a factor of 
Table 4. Spectral analysis results.

\begin{tabular}{|c|c|c|c|c|c|c|c|}
\hline $\begin{array}{l}\text { Phase } \\
( \pm 0.01)\end{array}$ & $\begin{array}{c}N_{\mathrm{H}} \\
10^{22} \mathrm{~cm}^{-2}\end{array}$ & $\begin{array}{l}\text { unabs } E W(\mathrm{Fe}) \\
\text { keV }\end{array}$ & $\begin{array}{c}\text { Fe line flux } \\
10^{-5} \mathrm{ph} \mathrm{cm}^{-1} \mathrm{~s}^{-1} \\
\end{array}$ & $\begin{array}{c}\text { Blackbody Luminosity } \\
10^{33} \mathrm{erg} \mathrm{s}^{-1} @ 8 \mathrm{kpc}\end{array}$ & $\begin{array}{c}I_{v}^{\text {cutoffpl }}(1 \mathrm{keV}) \\
10^{-3} \mathrm{ph} \mathrm{keV}^{-1} \mathrm{~cm}^{-2} \mathrm{~s}^{-1}\end{array}$ & $\begin{array}{c}F_{2-10 \mathrm{keV}}^{\text {unabs }} \\
10^{-10} \mathrm{erg} \mathrm{s}^{-1} \mathrm{~cm}^{-2}\end{array}$ & $\chi_{v}^{2}$ (d.o.f.) \\
\hline 0.01 & $17_{-9}^{+14}$ & $0.50 \pm 0.4$ & $0.34 \pm 0.21$ & $<0.88$ & $0.015_{-0.006}^{+0.009}$ & $0.0054 \pm 0.003$ & $1.34(23)$ \\
\hline 0.03 & $26_{-6}^{+9}$ & $0.81 \pm 0.4$ & $0.66_{-0.15}^{+0.12}$ & $<0.33$ & $0.018_{-0.005}^{-0.006}$ & $0.006(8) \pm 0.003$ & $2.6(17)$ \\
\hline 0.08 & $18_{-9}^{+0}$ & $1.1 \pm 0.6$ & $1.1 \pm 0.3$ & $<0.57$ & $0.023_{-0.007}^{+0.003}$ & $0.008(8) \pm 0.003$ & $1.06(11)$ \\
\hline 0.15 & $15.7 \pm 0.5$ & $0.04 \pm 0.01$ & $2.6_{-0.6}^{+0.8}$ & $1.6 \pm 1.4$ & $1.44 \pm 0.03$ & $0.48 \pm 0.02$ & $0.90(172)$ \\
\hline 0.27 & $9.4_{-0.3}^{+0.4}$ & $0.14 \pm 0.02$ & $14 \pm 02$ & $4.4_{-0.1}^{+0.3}$ & $2.29 \pm 0.05$ & $0.77 \pm 0.02$ & 1.05 (128) \\
\hline 0.37 & $12.1 \pm 0.3$ & $0.09 \pm 0.006$ & $16_{-2}^{+1}$ & $3.4_{-0.3}^{+0.1}$ & $3.86_{-0.06}^{+0.05}$ & $1.29 \pm 0.04$ & 1.09 (396) \\
\hline 0.40 & $19.1 \pm 0.5$ & $0.11 \pm 0.01$ & $11 \pm 1$ & $2.81_{-0.1}^{+0.06}$ & $2.26 \pm 0.05$ & $0.76 \pm 0.02$ & 1.45 (207) \\
\hline 0.65 & $24 \pm 1$ & $0.11 \pm 0.02$ & $7.6_{-1.5}^{+0.9}$ & $1.87_{-0.06}^{0.1}$ & $1.55_{-0.05}^{+0.06}$ & $0.52 \pm 0.02$ & $0.99(73)$ \\
\hline 0.79 & $23 \pm 3$ & $0.09 \pm 0.03$ & $2.5_{-07}^{+1.5}$ & $<1.48$ & $0.60 \pm 0.06$ & $0.20 \pm 0.04$ & $0.97(31)$ \\
\hline 0.91 & $89_{-14}^{+11}$ & $0.12 \pm 0.03$ & $3.2_{-0.7}^{+0.5}$ & $1.41_{-0.05}^{+0.1}$ & $0.6_{-0.1}^{+0.2}$ & $0.20 \pm 0.07$ & $0.82(33)$ \\
\hline
\end{tabular}

Notes. All free parameters are listed below together with the unabsorbed $2-10 \mathrm{keV}$ flux and $\chi_{v}^{2}$. All uncertainties have been calculated at $90 \%$ confidence level.

$\sim 200$ and remains stable at the level of $F_{\text {unabs }}^{2-10 \text { kev }} \approx(7 \pm 1) \times$ $10^{-13} \mathrm{erg} \mathrm{cm}^{-2} \mathrm{~s}^{-1}$.

The intrinsic absorbing column density (Fig. 4b) is persistently high $\left(\gtrsim 10^{23} \mathrm{~cm}^{-2}\right)$. Significant variations are detected for $\phi=0.2-0.4$ and close to the eclipse, reaching values of $N_{\mathrm{H}} \sim$ $9 \times 10^{23} \mathrm{~cm}^{-2}$.

The normalization of the blackbody component (Fig. 4c) does not show any variability, although the low energy part of the spectrum is poorly constrained. The normalization of the blackbody component is compatible with $\sim 10^{33} \mathrm{erg} \mathrm{s}^{-1}$ assuming a distance of $8 \mathrm{kpc}$. The very high intrinsic absorbing column density rules out the neutron star as the origin of the soft excess.

The flux and $E W$ of the $\mathrm{Fe} \mathrm{K} \alpha$ line are displayed in Figs. 4d and $4 \mathrm{e}$, respectively. Both components display significant variations indicating that the region emitting the line is at least partially obscured by the mass-donor star.

As the X-ray continuum illuminating the gas emitting the Fe fluorescent line cannot be measured during the eclipse, we calculated a corrected $\mathrm{Fe} \mathrm{K} \alpha E W$ by assuming a constant continuum flux of $1.8 \times 10^{-3} \mathrm{ph} \mathrm{keV}^{-1} \mathrm{~cm}^{-2} \mathrm{~s}^{-1}$ (Table 4).

\section{Discussion}

\subsection{Constraining the physical parameters of the system}

In the previous sections, we have derived an orbital solution (Table 3) yielding a mass function $f=4 \pi^{2}\left(\alpha_{x} \sin i\right)^{3} / G P_{\text {orb }}^{2}=$ $M_{\mathrm{OB}} \sin ^{3} i /(1+q)^{2} \approx 11.7 \pm 0.7 M_{\odot}$. This is consistent with a high mass X-ray binary system.

Adopting an inclination $i=90^{\circ}$, we infer a mass $M_{\mathrm{OB}} \sim$ $14 M_{\odot}$ for the donor star. Radial velocity observations showed that $q=M_{\mathrm{X}} / M_{\mathrm{OB}} \sim 0.1$ (Mason et al. 2010). Using an upper limit on the mass of $\sim 20 M_{\odot}$ (Thompson et al. 2007) constrains the inclination of the orbit $i>70^{\circ}$. Mason et al. (2010) estimated an inclination $i \approx 75^{\circ}-90^{\circ}$. Within this range, the mass of the donor star is constrained to be $M_{\mathrm{OB}} \approx 14-17 M_{\odot}$. The mass ratio and the donor mass imply a neutron star mass of $M_{\mathrm{NS}}=1.4-1.7 M_{\odot}$. The masses of both the donor star and the compact object are roughly similar (within a factor of 2) to that of Vela X-1 (Quaintrell et al. 2003; van Kerkwijk et al. 1995).

The separation of the system could be derived from the duration of the eclipse and the inclination (Joss \& Rappaport 1984). For the eclipse duration of $\Delta \phi \approx 0.18 \pm 0.02$ (i.e. 1.75 days), we can estimate the separation to be $\alpha_{x} \approx 1.7-1.8 R_{*}$. The
Roche lobe of the system is $R_{\mathrm{L}}=0.99-1.06 R_{*}$ assuming a synchronous rotation (Joss \& Rappaport 1984). This means that the system is very close to filling its Roche lobe and to forming an accretion disk, although no significant spin-up has been observed.

Using our VLT observations, Mason et al. (2009) performed near-IR spectroscopy of IGR J17252-3616 and concluded that the donor star is between B0-B5 I and B0-B1 Ia with an effective temperature $T_{\text {eff }}=22-28 k T$ and a stellar radius of $R_{*}=$ 22-36 $R_{\odot}$. On the basis of this spectroscopic determination, we obtain absolute numbers for the separation $\left(\alpha_{x} \approx 37-64 R_{\odot}\right)$ and the Roche lobe radius $\left(R_{\mathrm{L}} \approx 22-38 R_{\odot}\right)$.

The unabsorbed $2-10 \mathrm{keV}$ source flux is in the range $(0.2-1.3) \times 10^{-10} \mathrm{erg} \mathrm{s}^{-1} \mathrm{~cm}^{-2}$. Adopting a mean value of $(0.8 \pm 0.3) \times 10^{10} \mathrm{erg} \mathrm{s}^{-1} \mathrm{~cm}^{-2}$ and assuming a distance of $8 \mathrm{kpc}$ (Mason et al. 2009), the inferred 2-10 keV unabsorbed luminosity is $L_{\mathrm{X}} \sim 10^{36} \mathrm{erg} \mathrm{s}^{-1}$. Assuming accretion as the source of energy (where $L_{\mathrm{X}}=\epsilon \dot{M} c^{2}$ ), we can estimate a mass accretion rate of $\dot{M} \sim 10^{-9} M_{\odot} \mathrm{yr}^{-1}$, which is similar to that of Vela X-1 (Fürst et al. 2010).

During the eclipse, the X-ray luminosity drops by a factor of $\sim 200$ resulting in $L_{X} \sim 5 \times 10^{33} \mathrm{erg} \mathrm{s}^{-1}$. As OB stars emit in X-rays with a luminosity $L_{X} \sim 10^{31-32} \mathrm{erg} \mathrm{s}^{-1}$ (Güdel \& Nazé 2009), this emission is probably dominated by X-ray scattering in the stellar wind (Haberl 1991). Hickox et al. (2004) discussed the origins of the soft X-ray excesses of many types of accreting pulsars. It is likely that the fairly constant soft X-ray excess observed in IGR J17252-3616 is emitted by recombination lines (Schulz et al. 2002) in a region of the wind larger than the stellar radius (Watanabe et al. 2006).

For a spherically symmetric stellar wind, one would expect to have smooth and predictable variations in the absorbing column density along the orbit. In particular, observations along the same line of sight or symmetric when compared to the eclipse must result in different, respectively identical, column densities. Our observing strategy resulted in $N_{\mathrm{H}}(\phi=0.15) \approx N_{\mathrm{H}}(\phi=0.37)$, $N_{\mathrm{H}}(\phi=-0.35)>N_{\mathrm{H}}(\phi=0.37)$ and $N_{\mathrm{H}}(\phi=-0.35) \approx$ $N_{\mathrm{H}}(\phi=-0.21)$, ruling out a spherical geometry.

\subsection{Stellar wind structure}

We constructed a 3D model of the OB supergiant stellar wind to simulate the variability of the intrinsic column density $\left(N_{\mathrm{H}}\right)$ 


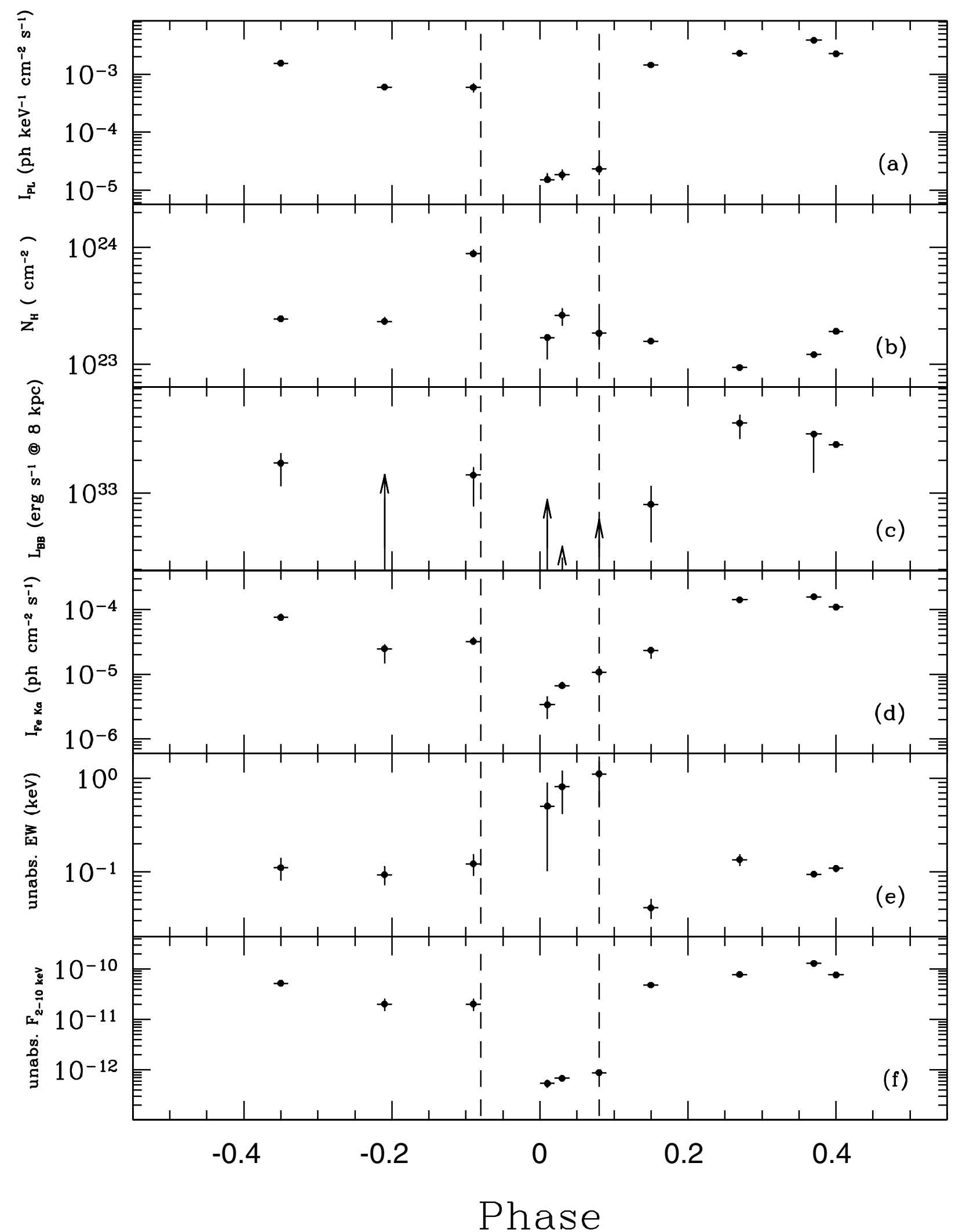

Fig. 4. Spectral variability along the orbit illustrated by a) the cut-off power-law normalization at $1 \mathrm{keV}$, b) the intrinsic hydrogen column density, c) the soft excess blackbody luminosity, assuming a distance of $8 \mathrm{kpc}$, d) the iron line flux, e) the iron line equivalent width calculated for the unabsorbed continuum, and f) the unabsorbed flux. Dashed vertical lines indicate the eclipse boundary. 
and the $\mathrm{Fe} \mathrm{K} \alpha$ line equivalent width $(E W)$, during the orbit, and compare them with the observations. We assumed a distance $D \approx 8 \mathrm{kpc}$ (Mason et al. 2009), a circular orbit ( $e=0$ ), and an edge-on geometry $\left(i=90^{\circ}\right)$.

\section{Variability of absorption along the orbit}

We first investigated the behavior of the intrinsic column density, $N_{\mathrm{H}}$, as a function of phase, to identify the structure of the wind during the orbit. We approximated the wind structure with two components, the unperturbed wind $\left(\rho_{\text {wind }}\right)$ and a tail-like hydrodynamic perturbation $\left(\rho_{\text {tail }}\right)$ related to the presence of the neutron star. Tail-like structures are expected for the photoionization and heating of the wind leading to strong shock formation and dense sheets of gas trailing the neutron star (Fransson $\&$ Fabian 1980). These shocks are produced by hydrodynamical simulations (Blondin et al. 1990) but produce a $N_{\mathrm{H}}$ of up to $\sim 10^{22} \mathrm{~cm}^{-2}$, which is too small to account for the variability observed in IGR J17252-3616.

The unperturbed stellar wind was modeled by assuming a standard wind profile (Castor et al. 1975)

$v(r)=v_{\infty}\left(1-\frac{R_{*}}{r}\right)^{\beta}$,

where $v(r)$ is the wind velocity at distance $r$ from the stellar center, $v_{\infty}$ is the terminal velocity of the wind, and $\beta$ is a parameter describing the wind gradient. The conservation of mass provides the radial density distribution of the stellar wind. The unperturbed stellar wind is a good approximation within the orbit of the neutron star. Hydrodynamical simulations (Blondin et al. 1990, 1991; Blondin 1994; Blondin \& Woo 1995; Mauche et al. 2008) of HMXB have shown that the wind can be highly disrupted by the neutron star beyond the orbit.

To estimate the terminal velocity of the unperturbed wind, we studied the $N_{\mathrm{H}}$ variability using three different sets of parameters (Fig. 5). The mass-loss rate and terminal velocity are constrained by the data to be in the range $\dot{M}_{\mathrm{w}} / v_{\infty} \sim(0.7-2) \times$ $10^{-16} M_{\odot} / \mathrm{km}(\beta$ has a very limited impact on the results, so we used 0.7).

The fraction of the wind captured by the neutron star could be estimated from the accretion radius $r_{\text {acc }}=2 G M_{\mathrm{X}} /\left(v_{\mathrm{orb}}^{2}+v^{2}\right) \sim$ $2 \times 10^{11} \mathrm{~cm}$ (where $v_{\text {orb }}=250 \mathrm{~km} \mathrm{~s}^{-1}$ is the orbital velocity) as $f \sim \pi r_{\text {acc }}^{2} / 4 \pi R_{\text {orb }}^{2} \sim 7.5 \times 10^{-4}$. The mass-loss rate is therefore $\dot{M}_{\mathrm{w}} \sim f^{-1} \dot{M} \approx 1.5 \times 10^{-6} M_{\odot} / \mathrm{yr}$ and the terminal velocity of the wind is constrained to be in the interval $v_{\infty} \sim 250-600 \mathrm{~km} / \mathrm{h}$.

In our simulation, we adopted a terminal velocity $v_{\infty}=$ $400 \mathrm{~km} \mathrm{~s}^{-1}$, a stellar radius $R_{*}=29 R_{\odot}$, a wind gradient $\beta=0.7$, and a mass loss rate $\dot{M}_{*}=1.35 \times 10^{-6} M_{\odot} \mathrm{yr}^{-1}$. The assumed, observed, and inferred model parameters are listed in Table 5. The variability of $N_{\mathrm{H}}$ along the orbit indicates that the unperturbed wind is adequate for phases $\phi \approx 0-0.35$. For $\phi \gtrsim 0.35$, $N_{\mathrm{H}}$ increases at a value of $\sim 2 \times 10^{23} \mathrm{~cm}^{-2}$. This indicates that a high density tail-like structure lies on one side of the orbit, trailing the neutron star. The tail-like component is still present up to $\phi \gtrsim 0.8$.

We assumed that the tail-like structure is created very close to the neutron star and opens up with distance. The density of the material inside the "tail" decreases with distance to ensure mass conservation. Its distribution follows a "horn"-like shape with a circular section. We adjusted the density of the tail-like structure to match the observations. The density distribution $\rho_{\text {wind }}+\rho_{\text {tail }}$ is displayed in Fig. 6. The supergiant is located at the center (black disk). The tail-like structure covers about half of the orbit.

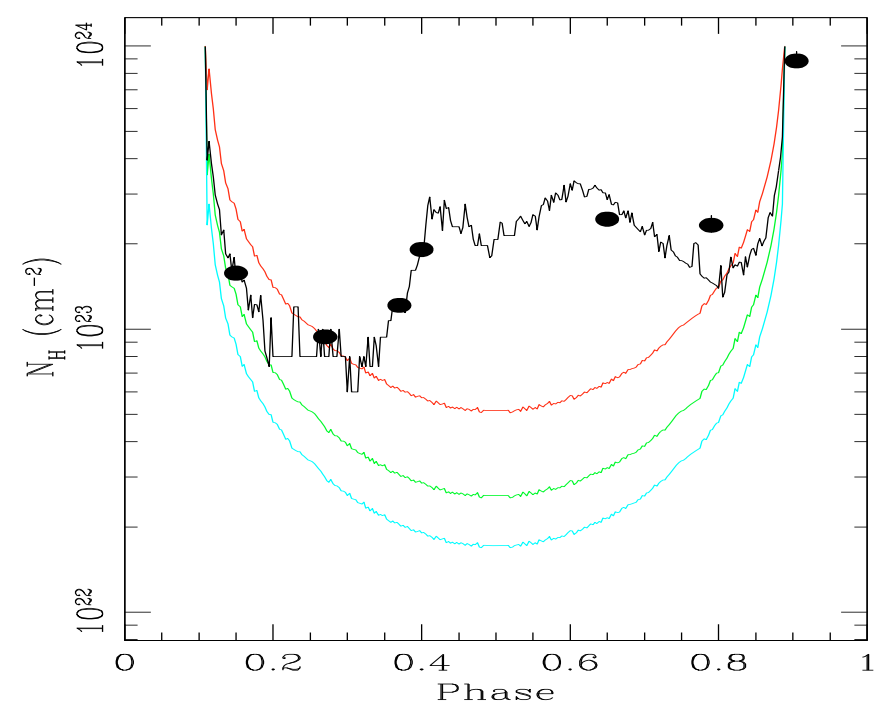

Fig. 5. Simulated $N_{\mathrm{H}}$ variations plotted together with the data. We illustrate three different smooth wind configurations, for $\dot{M} / v_{\infty}=0.7$ (cyan), 1 (green), and 2 (red) $\times 10^{-16} M_{\odot} / \mathrm{km}$, keeping all the other parameters fixed. The solid black line shows the total $N_{\mathrm{H}}$ consisting of the unperturbed stellar wind (green line) and the tail-like extended component. The observations during the eclipse have been omitted.

Table 5. Wind model parameters.

\begin{tabular}{lll}
\hline \hline Parameter & Value & Reference \\
\hline Observed & & \\
$q=M_{\mathrm{X}} / M_{\mathrm{OB}}$ & 0.1 & Mason et al. (2010) \\
$M_{\mathrm{OB}}$ & $15 M_{\odot}$ & Takeuchi et al. (1990) \\
$\alpha$ & $1.75 R_{*}$ & This work. \\
$\dot{M}$ & $10^{-9} M_{\odot} / \mathrm{yr}$ & This work. \\
\hline Assumed & & \\
$R_{*}$ & $29 R_{\odot}$ & \\
$M_{\mathrm{X}}$ & $1.5 M_{\odot}$ & \\
$i$ & $90^{\circ}$ & \\
$\beta$ & 0.7 & \\
\hline Inferred & & \\
$v_{\infty}$ & $400 \mathrm{~km} \mathrm{~s}^{-1}$ & \\
$\dot{M}_{\mathrm{w}}$ & $1.35 \times 10^{-6} M_{\odot} / \mathrm{yr}$ & \\
\hline
\end{tabular}

Figure 5 displays the simulated $N_{\mathrm{H}}$ variability from the above density distribution together with the observed data points. The data and the model shows that the tail-like perturbation is essential to understand the observed variations.

\section{Variability of the Fe $\mathrm{K} \alpha$ line during the orbit}

Assuming that the intrinsic X-ray flux is unaffected by the eclipse, the $\mathrm{Fe} \mathrm{K} \alpha$ equivalent width drops by a factor of $\sim 10$ during the eclipse in an orbital phase interval of $\sim 0.1$. This indicates that the radius of the region emitting $\mathrm{Fe} \mathrm{K} \alpha$ is smaller than half of the stellar radius $\left(<10^{12} \mathrm{~cm}\right)$ and far more compact than the tail structure responsible for the absorption variability profile.

Vela X-1 shows a similar behavior, which was interpreted as an emitting region of the size of (Ohashi et al. 1984), or even within (Endo et al. 2002) the accretion radius.

Outside the eclipse, the equivalent width of the $\mathrm{Fe} \mathrm{K} \alpha$ line is of the order of $100 \mathrm{eV}$. Following Matt (2002) and assuming a spherical transmission geometry, this corresponds to a column density of $N_{\mathrm{H}} \sim 2 \times 10^{23} \mathrm{~cm}^{-2}$. As this additional absorption 


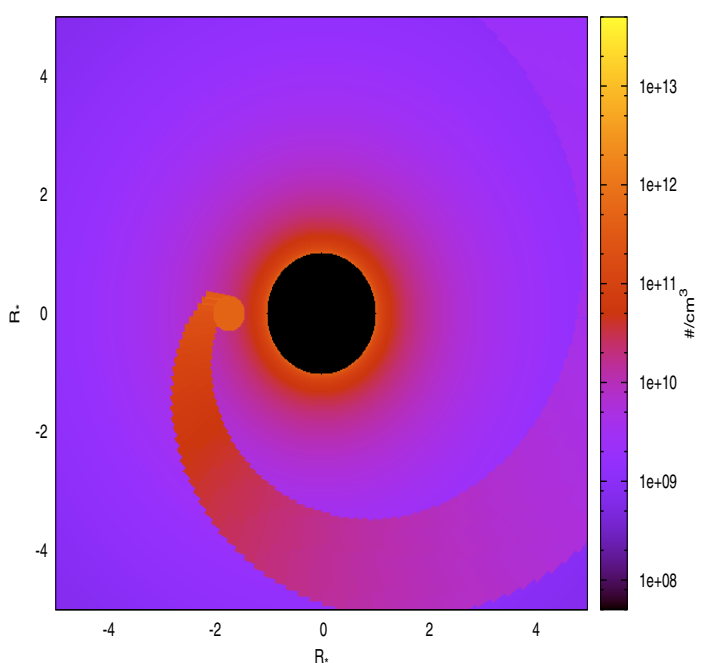

Fig. 6. Number density distribution in the plane of the orbit including a smooth wind and a tail-like perturbation. The black disk at the center represents the supergiant star.

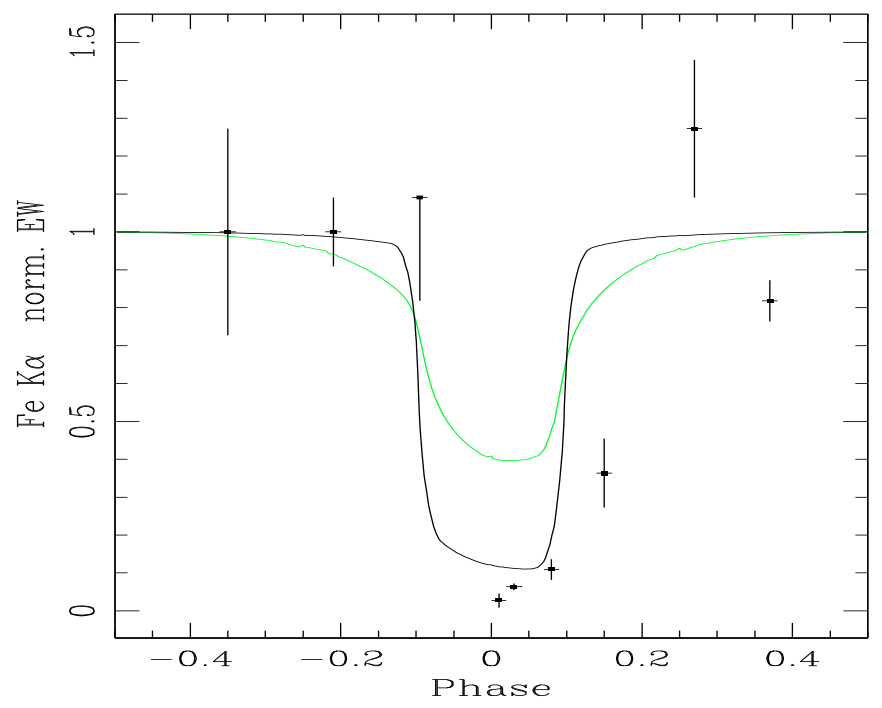

Fig. 7. Corrected unabsorbed $\mathrm{Fe} \mathrm{K} \alpha$ line equivalent width along the orbit. Green curve indicates the prediction of the hydrodynamical tail and the black curve the addition of the central cocoon.

is not observed, the region emitting $\mathrm{Fe} \mathrm{K} \alpha$ must be partially ionized.

An ionization parameter $\xi=L / n R^{2}$ in the range $10-300$ is required to fully ionize light elements contributing to the soft $\mathrm{X}$-ray absorption and keep an Fe $\mathrm{K} \alpha$ line at the energy of $6.4 \mathrm{keV}$ (Kallman et al. 2004; Kallman \& McCray 1982). The density of the Fe K $\alpha$ emitting region is therefore $\sim \xi^{-1} R_{12}{ }^{-2} \times 10^{12} \mathrm{~cm}^{-3}$, where $R_{12}$ is the distance from the neutron star in units of $10^{12} \mathrm{~cm}$. As $N_{\mathrm{H}}=n R \sim 2 \times 10^{23} \mathrm{~cm}^{-2}$, we have $\xi \sim 5 / R_{12}$. A dense cocoon is therefore needed around the neutron star with a size $0.5>R_{12}>0.02$ and a density $10^{11} \mathrm{~cm}^{-3}<n<$ $3 \times 10^{12} \mathrm{~cm}^{-3}$.

The majority of the $\mathrm{Fe} \mathrm{K} \alpha$ is formed in a region that is small enough to allow for pulsation of the $\mathrm{Fe} \mathrm{K} \alpha$ line, as observed in Cen X-3 (Day et al. 1993) and Her X-1 (Choi et al. 1994). We searched for such pulsations in our longest and almost uninterrupted observation (obsID 0405640801; $\phi=0.40$ ). Folded lightcurves were built in the energy bands $6.2-6.7 \mathrm{keV}$, $2-6.1 \mathrm{keV}$, and $6.8-10 \mathrm{keV}$ and resulted in a pulse fraction of

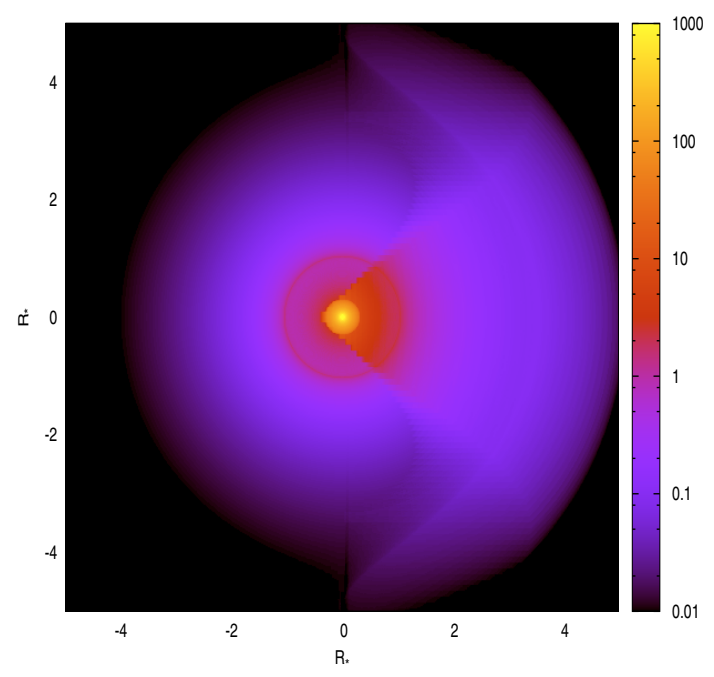

Fig. 8. Integrated $\mathrm{Fe} \mathrm{K} \alpha$ emissivity (relative units) centered on the neutron star at phase $\phi=0.5$. The smooth circular halo shows the rim of the supergiant star. The tail structure can be observed on the right.

$49 \pm 5 \%, 58 \pm 3 \%$, and $57 \pm 4 \%$, respectively. The ratio of the line flux to the continuum in the energy range $6.2-6.7 \mathrm{keV}$ is $\sim 0.25$. Assuming that the line is not pulsed, we infer an $\mathrm{Fe} \mathrm{K} \alpha$ pulse fraction of $\sim 50 \%$, which is in good agreement with the above measurement. Weak Fe K $\alpha$ pulsation can be explained if the cocoon is isotropic.

To match the observed column density variability, the radius and the density of the inner region of the tail structure were set to $4 \times 10^{11} \mathrm{~cm}$ and $3 \times 10^{11} \mathrm{~cm}^{-3}$, respectively. It is therefore very likely that the dense cocoon corresponds to the inner and ionized region of the hydrodynamical tail. We thus added this partially ionized cocoon in our simulations, using a density of $3 \times 10^{11} \mathrm{~cm}^{-3}$ within a radius of $\sim 6 \times 10^{11} \mathrm{~cm} \sim 3 R_{\mathrm{acc}}$. The Fe K $\alpha$ emissivity map (Fig. 8) was calculated by applying an illuminating radiation field $\left(\sim 1 / r^{2}\right)$ to the density distribution. Figure 7 displays the resulting simulated profile of the $\mathrm{Fe} \mathrm{K} \alpha$ equivalent width together with the observed data. The green curve shows the variations in the $\mathrm{Fe} \mathrm{K} \alpha$ equivalent width expected from the wind density profile excluding the central cocoon, which obviously could not reproduce the data. The black curve accounts for the dense central cocoon. The exact profile of the eclipse is related to the size and density profile of the cocoon. No effort has been made to obtain an exact match to the data.

The ionized cocoon is expected to produce an iron K-edge at $\sim 7.8 \mathrm{keV}$. For a column density of $N_{\mathrm{H}} \sim 2 \times 10^{23} \mathrm{~cm}^{-1}$, its optical depth $\tau \sim 0.2$ (Kallman et al. 2004) remains difficult to detect. Even our observation at an orbital phase 0.15 (the best candidate for the detection of the ionised edge) does not have enough signal.

The mass of the tail-like structure $M_{\text {tail }} \sim 10^{-8} M_{\odot}$ can be accumulated in $t_{\text {tail }}=M_{\text {tail }} / \dot{M}$, where $\dot{M}=\left(\pi r_{\text {eff }}^{2} / 4 \pi R_{\text {orb }}^{2}\right) \dot{M}_{\mathrm{w}}$ and $r_{\text {eff }}$ is an effective radius for the funneling of the wind in the tail. For a tail accumulation timescale $\left(t_{\text {tail }}\right)$ comparable to the orbital period of ( $\sim 10$ days), this effective radius is $12 R_{\text {acc }}$.

The orientation of the tail-like structure depends on the wind and orbital velocities. The angle between the wind velocity and the orbital velocity is given by $\tan (\alpha)=v_{\text {orb }} / v$. The tail obtained in our simulations is tilted by $\alpha \sim 80^{\circ}$. This corresponds to $v \sim$ $0.2 v_{\text {orb }} \approx 50 \mathrm{~km} \mathrm{~s}^{-1}$, which is lower than $v\left(R_{\text {orb }}\right) \approx 250 \mathrm{~km} \mathrm{~s}^{-1}$ because of the ionization of the stellar wind in the vicinity of the neutron star. 


\section{Conclusions}

We have presented the analysis of an observing campaign performed with XMM-Newton on the persistently absorbed sgHMXB IGR J17252-3616. Nine observations have been performed over about four weeks, distributed across various orbital phases. Three of them were scheduled during the eclipse of the neutron star by the companion star.

We first refined the orbital solution, using in addition archival INTEGRAL and RXTE data and found an orbital period of $9.742 \mathrm{~d}$ and a projected orbital radius of $101 \pm 2$ lt-s. The pulsar spin period varies between 414.3 and $413.8 \mathrm{~s}$ during the observing campaign.

The X-ray spectrum $(0.2-10 \mathrm{keV})$, which varies during the orbit, was successfully fitted using an absorbed cut-off powerlaw continuum, a soft excess, and a gaussian emission line. The soft excess, modeled with a black body, remained constant.

The continuum component varies in intensity (a measure of the instantaneous accretion rate) but displays a constant spectral shape, as usually observed in accreting pulsars.

The absorbing column density and the $\mathrm{Fe} \mathrm{K} \alpha$ emission line show remarkable variations. The column density, always above $10^{23} \mathrm{~cm}^{-2}$, increases towards $10^{24} \mathrm{~cm}^{-2}$ close to the eclipse, as expected for a spherically symmetric wind. The wind velocity is unusually small close to $v_{\infty}=400 \mathrm{~km} \mathrm{~s}^{-1}$. An additional excess of absorption of $2 \times 10^{23} \mathrm{~cm}^{-2}$ is observed for orbital phases $\phi>0.3$, which is found to represent a hydrodynamical tail trailing the neutron star.

During the eclipse, the equivalent width of the $\mathrm{Fe} \mathrm{K} \alpha$ line drops by a factor $>10$ indicating that most of the line is emitted in a cocoon surrounding the pulsar, with a size of a few accretion radii. This cocoon is ionized and corresponds to the inner region of the hydrodynamical tail.

The parameters of the IGR J17252-3616 are very similar to these of Vela X-1, except for the smaller wind velocity. We argue that the persistently large absorption column density is related to the hydrodynamical tail, which has been strengthened by the low wind velocity. The tail is a persistent structure dissolving on a timescale comparable to the orbital period.

Our interpretation can be tested using numerical hydrodynamical simulations and high resolution optical/infrared spectroscopy. If confirmed, it may turn out that half of the persistent sgHMXB have stellar wind speeds several times lower than usually measured.

\section{References}

Arnaud, K. A. 1996, in Astronomical Data Analysis Software and Systems V, ed. G. H. Jacoby, \& J. Barnes, ASP Conf. Ser., 101, 17
Blondin, J. M. 1994, ApJ, 435, 756

Blondin, J. M., \& Woo, J. W. 1995, ApJ, 445, 889

Blondin, J. M., Kallman, T. R., Fryxell, B. A., \& Taam, R. E. 1990, ApJ, 356, 591

Blondin, J. M., Stevens, I. R., \& Kallman, T. R. 1991, ApJ, 371, 684

Castor, J. I., Abbott, D. C., \& Klein, R. I. 1975, ApJ, 195, 157

Charles, P. A., \& Coe, M. J. 2006, Optical, ultraviolet and infrared observations of X-ray binaries, ed. W. H. G. Lewin, \& M. van der Klis, 215

Chaty, S., Rahoui, F., Foellmi, C., et al. 2008, A\&A, 484, 783

Choi, C. S., Nagase, F., Makino, F., et al. 1994, ApJ, 437, 449 Corbet, R. H. D. 1986, MNRAS, 220, 1047

Day, C. S. R., Nagase, F., Asai, K., \& Takeshima, T. 1993, ApJ, 408, 656

Dickey, J. M., \& Lockman, F. J. 1990, ARA\&A, 28, 215

Endo, T., Ishida, M., Masai, K., et al. 2002, ApJ, 574, 879

Fransson, C., \& Fabian, A. C. 1980, A\&A, 87, 102

Fürst, F., Kreykenbohm, I., Pottschmidt, K., et al. 2010, A\&A, 519, A37

Güdel, M., \& Nazé, Y. 2009, A\&ARv, 17, 309

Haberl, F. 1991, A\&A, 252, 272

Hickox, R. C., Narayan, R., \& Kallman, T. R. 2004, ApJ, 614, 881

Jansen, F., Lumb, D., Altieri, B., et al. 2001, A\&A, 365, L1

Joss, P. C., \& Rappaport, S. A. 1984, ARA\&A, 22, 537

Kallman, T. R., \& McCray, R. 1982, ApJS, 50, 263

Kallman, T. R., Palmeri, P., Bautista, M. A., Mendoza, C., \& Krolik, J. H. 2004 ApJS, 155, 675

Lebrun, F., Leray, J. P., Lavocat, P., et al. 2003, A\&A, 411, L141

Levine, A. M., Rappaport, S., Remillard, R., \& Savcheva, A. 2004, ApJ, 617, 1284

Liu, Q. Z., van Paradijs, J., \& van den Heuvel, E. P. J. 2006, A\&A, 455, 1165

Mason, A. B., Clark, J. S., Norton, A. J., Negueruela, I., \& Roche, P. 2009, A\&A, 505,281

Mason, A. B., Norton, A. J., Clark, J. S., Negueruela, I., \& Roche, P. 2010, A\&A, 519, A79

Matt, G. 2002, MNRAS, 337, 147

Mauche, C. W., Liedahl, D. A., Akiyama, S., \& Plewa, T. 2008, in Am. Inst. Phys. Conf. Ser., ed. M. Axelsson, 1054, 3

Ohashi, T., Inoue, H., Koyama, K., et al. 1984, PASJ, 36, 699

Press, W. H., \& Rybicki, G. B. 1989, ApJ, 338, 277

Quaintrell, H., Norton, A. J., Ash, T. D. C., et al. 2003, A\&A, 401, 313

Rahoui, F., Chaty, S., Lagage, P., \& Pantin, E. 2008, A\&A, 484, 801

Schulz, N. S., Canizares, C. R., Lee, J. C., \& Sako, M. 2002, ApJ, 564, L21

Strüder, L., Briel, U., Dennerl, K., et al. 2001, A\&A, 365, L18

Takeuchi, Y., Koyama, K., \& Warwick, R. S. 1990, PASJ, 42, 287

Tawara, Y., Yamauchi, S., Awaki, H., et al. 1989, Astron. Soc. Japan, 41, 473

Thompson, T. W. J., Tomsick, J. A., in 't Zand, J. J. M., Rothschild, R. E., \& Walter, R. 2007, ApJ, 661, 447

van Kerkwijk, M. H., van Paradijs, J., Zuiderwijk, E. J., et al. 1995, A\&A, 303, 483

Walter, R., Bodaghee, A., Barlow, E. J., et al. 2004, The Astronomer's Telegram, 229, 1

Walter, R., Zurita Heras, J., Bassani, L., et al. 2006, A\&A, 453, 133

Warwick, R. S., Norton, A. J., Turner, M. J. L., Watson, M. G., \& Willingale, R. 1988, MNRAS, 232, 551

Watanabe, S., Sako, M., Ishida, M., et al. 2006, ApJ, 651, 421

White, N. E. 1989, A\&ARv, 1, 85

White, N. E., Nagase, F., \& Parmar, A. N. 1995, in X-ray Binaries, ed. W. H. G. Lewin, J. van Paradijs, \& E. P. J. van den Heuvel, 1

Winkler, C., Courvoisier, T., Di Cocco, G., et al. 2003, A\&A, 411, L1

Zurita Heras, J. A., de Cesare, G., Walter, R., et al. 2006, A\&A, 448, 261 